\title{
STATISTICAL EVIDENCE FOR THE EXISTENCE OF ALFVÉNIC TURBULENCE IN SOLAR CORONAL LOOPS
}

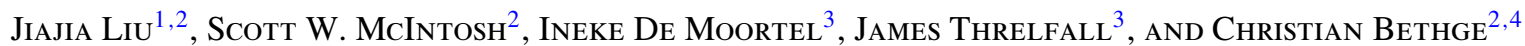 \\ ${ }^{1}$ Earth and Space Science School, University of Science and Technology of China, No. 96, JinZhai Road, Hefei, China; 1jj128@ mail.ustc.edu.cn \\ ${ }^{2}$ High Altitude Observatory, National Center for Atmospheric Research, P.O. Box 3000, Boulder, CO 80307, USA \\ ${ }^{3}$ School of Mathematics and Statistics, University of St Andrews, St Andrews, Fife KY16 9SS, UK \\ ${ }^{4}$ Kiepenheuer Institute for Solar Physics, Schöneckstraße 6, D-79104 Freiburg, Germany \\ Received 2014 May 27; accepted 2014 September 25; published 2014 November 18
}

\begin{abstract}
Recent observations have demonstrated that waves capable of carrying large amounts of energy are ubiquitous throughout the solar corona. However, the question of how this wave energy is dissipated (on which timescales and length scales) and released into the plasma remains largely unanswered. Both analytic and numerical models have previously shown that Alfvénic turbulence may play a key role not only in the generation of the fast solar wind, but in the heating of coronal loops. In an effort to bridge the gap between theory and observations, we expand on a recent study by analyzing 37 clearly isolated coronal loops using data from the Coronal Multi-channel Polarimeter instrument. We observe Alfvénic perturbations with phase speeds which range from 250 to $750 \mathrm{~km} \mathrm{~s}^{-1}$ and periods from 140 to $270 \mathrm{~s}$ for the chosen loops. While excesses of high-frequency wave power are observed near the apex of some loops (tentatively supporting the onset of Alfvénic turbulence), we show that this excess depends on loop length and the wavelength of the observed oscillations. In deriving a proportional relationship between the loop length/wavelength ratio and the enhanced wave power at the loop apex, and from the analysis of the line widths associated with these loops, our findings are supportive of the existence of Alfvénic turbulence in coronal loops.
\end{abstract}

Key words: Sun: corona - waves

Online-only material: color figures

\section{INTRODUCTION}

Recent advances in the field of ground- and space-based observations of the solar corona have revealed the prevalence of oscillatory/wave-like phenomena across a wide range of structures pervading the solar atmosphere (e.g., De Moortel \& Nakariakov 2012). The growing evidence of wave propagation (and dissipation) has lead to a resurgence of interest in waves and their contribution to both the heating of the solar atmosphere and the generation of the fast solar wind (e.g., Tomczyk et al. 2007; McIntosh et al. 2011; Parnell \& De Moortel 2012). Since acoustic wave heating is thought to be efficient only over relatively low heights (Osterbrock 1961), magnetoacoustic (MA) waves and/or Alfvén(ic) waves have instead been regarded as likely candidates for oscillatory/wave-like phenomena observed along coronal structures (see, e.g., Parker 1991; Suzuki \& Inutsuka 2005; Nakariakov \& Verwichte 2005; McIntosh et al. 2011; McIntosh 2012; De Moortel \& Nakariakov 2012).

Both fast-MA kink waves and/or Alfvén waves may generate observational signatures which are typically seen as propagating transverse oscillatory displacements of the local parent structure. Such signatures have been observed along many different types of structures, including (but not limited to) coronal loops (e.g., Ulmschneider \& Musielak 1990; Aschwanden et al. 1999; Tomczyk et al. 2007; Threlfall et al. 2013; Morton \& McLaughlin 2013), jets (Cirtain et al. 2007), spicules (De Pontieu et al. 2007; He et al. 2009), prominences (e.g., Okamoto et al. 2007), and coronal rain (e.g., Antolin \& Verwichte 2011). However, interpretation of these and other observations in terms of a pure fast-MA kink or Alfvén wave mode alone requires additional information about the local geometry/environment of the parent structure; a substantial (and ongoing) debate regarding the underlying nature of these observations has arisen (see, e.g., Erdélyi \& Fedun 2007; Van Doorsselaere et al. 2008; De Moortel \& Nakariakov 2012).

Recent numerical simulations have shown that generic transverse footpoint displacements generate propagating oscillatory displacements which are composed of coupled kink-Alfvén modes (e.g., Hood et al. 2013; Pascoe et al. 2013; Terradas et al. 2010, and references therein); the term "Alfvénic" has come to describe this inherently coupled wave mode, which cannot be entirely described by a single wave mode alone (e.g., McIntosh et al. 2011). Estimates of the energy flux carried by these waves may be sufficient to balance the energy budget of (for example) the quiet Sun (e.g., De Moortel \& Pascoe 2012; McIntosh \& De Pontieu 2012; Goossens et al. 2013), and hence could form a crucial part of the energy transport into the corona and solar wind. While these waves are certainly capable of carrying the energy over large distances within the corona, one vital piece of the puzzle remains elusive; the mechanism by which energy is extracted from the waves.

Damping mechanisms are often underpinned by an energy cascade from large to small scales. The Alfvénic wave mode allows access to several such processes. For example, numerical simulations that highlight wave mode coupling (Pascoe et al. 2013) show that energy is transferred from footprint-driven (bulk) transverse motion to an azimuthal component, which can then phase-mix within a relatively small boundary layer of strong inhomogeneity. While this does lead to wave damping, it does so only in narrow regions that are (at present) beyond the resolution limit of current observations (however, it is possible that future high spatial and temporal resolution instruments, for example, those on the Daniel K. Inouye Solar Telescope, may be able to shed some light on such layers, and their role in the energy transport). Transferring energy to smaller scales could lead to Alfvénic turbulence, and has been shown to be 

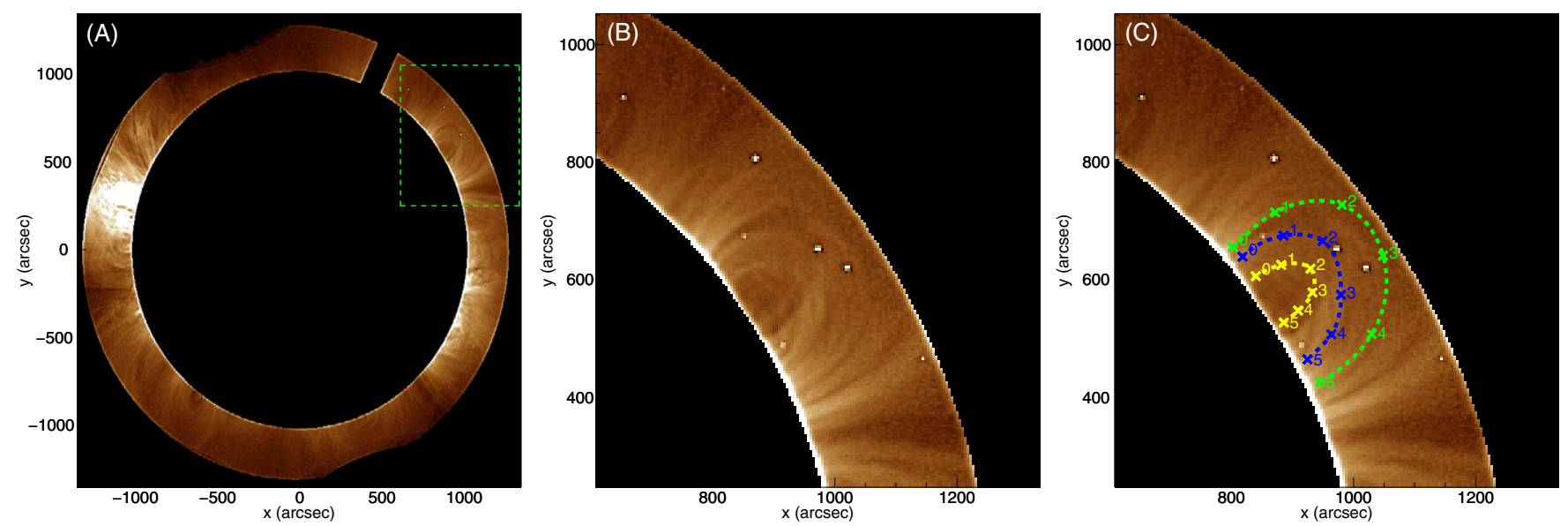

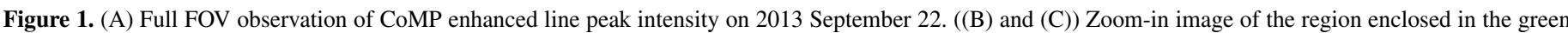

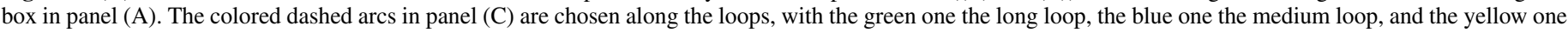
the short loop.

(A color version of this figure is available in the online journal.)

able to account for the acceleration of the fast solar wind (e.g., Parker 1991; Oughton et al. 2001; Cranmer \& van Ballegooijen 2005; Verdini et al. 2010) and heating of coronal loops (e.g., van Ballegooijen et al. 2011; Asgari-Targhi \& van Ballegooijen 2012). While a great deal of progress on this issue has been made using both analytical and numerical models (particularly with regard to solar wind acceleration), it is also important to relate these models to observations. The recent work by De Moortel et al. (2014), which motivated this paper, has shown the tentative evidence for the onset of Alfvénic turbulence in a trans-equatorial coronal loop, by exploring the novel "excess of high-frequency fast Fourier transform (FFT) power" (hereafter referred as the "EHFF") phenomenon near the loop apex.

Following De Moortel et al. (2014), we present a detailed statistical analysis of 37 clearly isolated coronal loops observed in the field-of-view (FOV) of the Coronal Multi-channel Polarimeter (CoMP) instrument (Tomczyk et al. 2008). This analysis suggests that the extra high-frequency part of the power spectrum (previously described as tentative evidence for the onset of Alfvénic turbulence) is particularly prevalent for coronal loops with length 3.0 times longer than the characteristic wavelength of the propagating waves. Our paper is organized as follows. In Section 2 we describe the instrument and data used in the analysis, while a detailed study of three coronal loops is presented as an example in Section 3, followed by a statistical survey of all our chosen loops in Section 4. A discussion of our results can be found in Section 5 before presenting our conclusions in Section 6.

\section{INSTRUMENT AND DATA}

The CoMP (Tomczyk et al. 2008) is a combination polarimeter and narrowband tunable filter that can measure the complete polarization state in the vicinity of the $10747 \AA$ and $10798 \AA$ Fe XIII coronal emission lines. It was deployed behind the $20 \mathrm{~cm}$ aperture Coronal One Shot (COS) coronagraph (Smartt et al. 1981) and is now mounted on the spar at the Mauna Loa Solar Observatory. CoMP is comprised of (1) an occulting disk, located at the focus of the COS, that blocks the light from the solar disk; (2) a lens that collimates the solar image; (3) a filter wheel holding three order-blocking filters corresponding to each of the three observable emission line regions; (4) the polarimeter/ tunable filter package; (5) a re-imaging lens that forms the final solar image; and (6) a $1024 \times 1024$ pixel $\mathrm{HgCdTe}$ infrared detector array. The CoMP filter is a four-stage, wide-field calcite birefringent filter with a bandwidth of $1.3 \AA$ (an instrumental width of $21 \mathrm{~km} \mathrm{~s}^{-1}$ ) and is tuned in wavelength by four liquid crystal variable retarders and has a full FOV of $2.8 R_{\odot}$ at a spatial sampling of 4.5 . The data studied in this paper are the "Dynamics 3" data which take three wavelength positions at the $10747 \AA$ Fe XIII line and cadence of $30 \mathrm{~s}$. All of the data analyzed below are openly available on the CoMP Webpage (http://www.cosmo.ucar.edu/COMP.html).

The reduced CoMP FITS data contain four components: line peak intensity, Doppler velocity, line width, and the enhanced intensity. We have found 46 isolated (without significant line-ofsight complexity) bright coronal loops sets for study that can be grossly grouped as belonging to coronal cavities, active regions and trans-equatorial systems. We then compared these loops observed in the CoMP enhanced line peak intensity images with those tracked through a "wave-tracking" method, which employs the wave-propagation-angle map generated by a crosscorrelation method on the Doppler velocity images (McIntosh et al. 2008). Nine of these loops have been discarded due to unsatisfactory results from the wave-tracking method (errors in phase speeds exceeding $100 \mathrm{~km} \mathrm{~s}^{-1}$ ), which may be a result of line-of-sight superposition effects. For the remaining loops we use CoMP data with less than 3 missing frames with at least 90 minutes of continuous observation. Any data gaps are filled by linear interpolation using the preceding and following images. As in Threlfall et al. (2013), we select six points along a given coronal loop to define an arc using spline interpolation (see, for example, Figure 1(B)). The coordinates of the arc are subsequently resampled to be equally spaced, where the spacing is chosen to be the CoMP pixel size of 4".5 (3.24 Mm) for simplicity. For every position along the loop, data are also sampled along a $\sim 20 \mathrm{Mm}$ perpendicular cut, again spaced by the 4..5 (3.24 Mm) CoMP resolution and hence building up a grid of perpendicular cuts centered on the arc.

\section{EXAMPLE: THE CAVITY LOOPS OF 2013 SEPTEMBER 22}

In this section, we present a detailed analysis of a (long) coronal loop that was part of a coronal cavity on 2013 September 22 

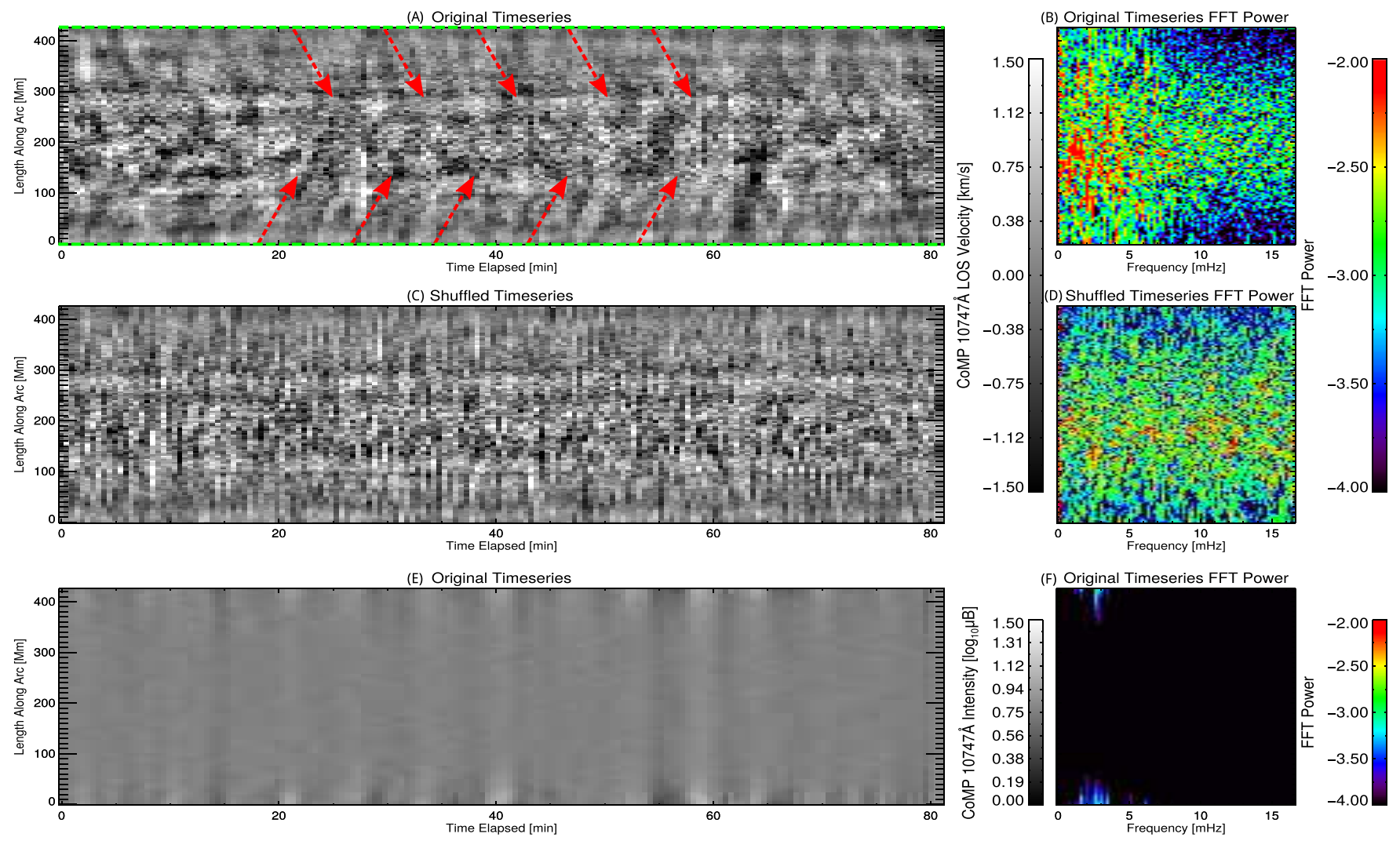

Figure 2. ((A) and (C)) Time-distance plots of Doppler velocity along the arc highlighted by the green curve in Figure 1(C), based on the original and reshuffled time series, respectively. Perturbations with phase speed around $640 \mathrm{~km} \mathrm{~s}^{-1}$ are indicated by red dashed arrows in panel (A). Two green dashed horizontal lines represent the footpoints of the arc. ((B) and (D)) Corresponding FFT power spectra of panels (A) and (C) as functions of distance and frequency. FFT power in panel (B) for the original time series and panel (D) for the reshuffled time series. ((E) and (F)) Corresponding time-distance plot of line peak intensity along the arc based on the original time series and its associated FFT power spectrum.

(A color version of this figure is available in the online journal.)

as an example (see De Moortel et al. 2014), and then compare the results with those from two shorter loops in the same cavity.

Figure 1(A) shows the CoMP enhanced line peak intensity for the full FOV on 2013 September 22. The green box outlines the region of the cavity on the northwest limb shown where Figures 1(B) and (C) show a close-up view of the cavity region. In this region we have identified three clearly isolated loops, outlined in green (the long loop), blue (the medium loop), and yellow (the short loop), respectively, and with position angle (P.A.) of $57^{\circ}$, where P.A. is measured in degrees from solar north to the apex of the loop.

Let us start with the analysis of the long (green) loop. As described in Section 2, we repeat the spline-fitting procedure of Threlfall et al. (2013) by choosing six spline points along the loop, shown as six green stars labeled from 0 (footpoint 1) to 5 (footpoint 2) in Figure 1(C). These spline points are used to generate an arc, shown in green in Figure 1(C), with the spacing along the arc equal to the CoMP resolution of 4".5 (3.24 Mm). By integrating the distance between pixels along the arc, the length of the arc is calculated to be $\sim 305^{\prime \prime}(\sim 420 \mathrm{Mm})$. We should point out, however, that the length of the arc does not equal the real (physical) length of the loop due to (1) projection effects and (2) the fact that the spline points (0) and (5) are not located at the exact footpoints of the loop. To compensate for the latter effect, we add the distance from the footpoints to the solar limb to the length of the arc, giving a total length of $452 \mathrm{Mm}$. Projection effects have not been taken into account as it is difficult to exactly identify the corresponding object in STEREO images for a loop found at the limb of CoMP.
Figure 2(A) shows the time-distance plot of the CoMP Doppler velocity, along the arc highlighted in Figure 1(C) by the dashed green line. A clear "herringbone" pattern of perturbations (red dashed arrows in Figure 2(A)), originating from both of the loop footpoints (green dashed lines in Figure 2(A)), is visible in the time-distance diagram (similar to the example analyzed by De Moortel et al. 2014). These perturbations are not generally seen to travel all the way along the loop as they rarely appear to reach the opposite footpoint - a tantalizing signature that the waves are changing in passage. The time-distance pattern is less distinct near the loop apex, possibly due to the interaction of the perturbations traveling upward from the opposite footpoint.

The phase speed of the perturbations is computed using the cross-correlation method developed by Tomczyk \& McIntosh (2009). We cross-correlate the time series at each position along the arc with the time series at the midpoint of the arc (i.e., the apex of the loop). The peak of the cross-correlation function is then fitted with a parabola such that lag or lead time at each point along the arc is returned. We then fit the lag/lead times versus the distance along the loop with a straight line-the phase speed (and the associated error in the phase speed) of the propagating perturbations are the gradient of this line. When waves are counter propagating this technique gives rise to anomalously high phase speeds (see Tomczyk \& McIntosh 2009 for more details). Therefore, to obtain more accurate phase speeds, we use the technique of Tomczyk \& McIntosh (2009) to identify the phase speeds of waves moving in either direction along the loop: pro-retro-grade filtering is done by masking the 
positive/negative frequency halves of the $k-\omega$ diagram generated from the FFT of the original time-distance plot, and then performing the inverse transform to construct two space time plots, one for each direction of propagation. The crosscorrelation phase speed method is applied to each to yield the (mean) phase speed of the wave on that arc, where the two propagation directions mostly show very similar phase speeds.

By averaging the phase speeds obtained from the filtered time-distance plots employing the cross-correlation method described above, the phase speed of perturbations (waves) propagating along the loop is estimated at $640( \pm 34) \mathrm{km} \mathrm{s}^{-1}$ (red dashed arrows in Figure 2(A)). It is substantially larger than the (estimated) local sound speed of a cavity $\left(\sim 100 \mathrm{~km} \mathrm{~s}^{-1}\right.$; Liu et al. 2012). As in previous studies (e.g., Tomczyk et al. 2007; Tomczyk \& McIntosh 2009; Threlfall et al. 2013; De Moortel et al. 2014), we found little evidence of simultaneous intensity variations (Figure 2(E)), indicating that the traveling perturbations are largely non-compressive (Alfvénic) in nature.

Figure 2(B) shows the logarithm of the FFT power of the time-distance data in Figure 2(A) as a function of frequency and distance along the arc shown in Figure 1. Detailed investigation of the FFT power diagram reveals that (1) most of the power is concentrated at the lower frequency range, as shown with red colors in the left portion of the diagram; (2) the FFT power at the apex (middle part along the $y$-axis direction) is consistently higher than at the footpoints (edge regions with maximum and minimum $y$ value) for all frequencies. This higher power could be caused by linear superposition of the perturbations propagating upward from both footpoints and/ or the effect of gravitational stratification, which leads to the growth of velocity amplitudes with height (see, e.g., Wright \& Garman 1998). As a baseline comparison, we perform a random reshuffle of the original time series (De Moortel et al. 2014). The corresponding time-distance plot and FFT power spectrum are shown in Figures 2(C) and (D). It is clear that there is no longer any evidence of the characteristic herringbone pattern of perturbations. The corresponding FFT power spectrum is almost uniform-power is equally distributed over all frequencies.

To investigate whether the superposition and/or gravitational stratification effects could fully account for the growth of the FFT power at the apex, we average the FFT power across the low- (>8 minutes), medium- (between 3 and 8 minutes), and high-frequency ( $<3$ minutes) ranges (hereafter referred to as LF, $\mathrm{MF}$, and $\mathrm{HF}$, respectively). The resulting averaged power for the three frequency ranges as a function of distance along the arc is shown in Figure 3(A). The curves have been smoothed to remove the smallest scale variations and have been normalized to their own averages to allow easy comparison. It is clear from Figure 3(A) that the FFT power for the three frequency ranges grows at the apex. However, the growth rate for these three curves appears to differ - the FFT power of the higher frequencies grows faster than that of the lower frequencies. Following the estimate of De Moortel et al. (2014), the wave amplitude could increase by a maximum factor of 3.4 at the loop apex, using linear superposition (a maximum factor of 2) and an $e^{z_{\text {apex }} /(4 H)} \approx 1.7$ gravitational growth (see, e.g., Wright \& Garman 1998) with the gravitational scale height $H=75 \mathrm{Mm}$ and the height of the loop apex $z_{\text {apex }}=160 \mathrm{Mm}$ for this particular loop. Figure 3(A) shows that the FFT power for the LF and MF parts is about 1.36 higher at the apex than at footpoints, indicating a $\sqrt{1.36} \approx 1.17$ growth in wave amplitude (as the FFT power scales as the square of the amplitude), which corresponds to a damping of up to $65 \%$ compared to the ideal non-damping estimation (as 1.17 is about $35 \%$ of 3.4 , the maximum possible growth rate). On the other hand, the damping of the HF part is estimated to be roughly $55 \%$, less than that of the MF and LF parts, implying the presence of an additional effect other than the linear superposition and gravitational stratification. The results for the randomly shuffled time series (Figure 3(B)) show similar growth rates for the three frequency ranges.

Figure 3(C) shows the logarithm of the averaged FFT power over distance as a function of frequency. To remove the influence of linear superposition and gravitational stratification effects, the FFT power at each position along the loop is divided by the total FFT power over the whole frequency range at that position. The two dashed black curves show the FFT power at the two footpoint regions (the first and last 20 points of the arc) and the solid curve is their average. The solid red curve is the FFT power around the apex (the middle 20 points of the arc). The FFT power decreases with frequency at the footpoints as well as at the apex. However, a detailed comparison of the two solid curves reveals that the power at the apex (red curve) is less than or at most equal to that of the footpoints (black curve) in the LF and MF ranges, but becomes higher in the HF range. The ratio of the power at the apex and footpoints is about 1.01 and 0.80 , in the LF and MF frequency ranges, respectively. However, the ratio grows to about 1.81 in the HF range, implying an "excess" of HF power. The randomly shuffled time series on the other hand (Figure 3(D)) shows an even distribution of the FFT power as a function of frequency for the three different regions. To quantify the excess HF FFT power, we define a variable "ratio difference" (RD) which represents how much the ratio $(R)$ between the power at the apex and footpoints grows at the $\mathrm{HF}$ range with respect to the $\mathrm{LF}$ and $\mathrm{MF}$ ranges, i.e.,

$$
\mathrm{RD}=\frac{R_{\mathrm{HF}}-\left(R_{\mathrm{LF}}+R_{\mathrm{MF}}\right) / 2}{\left(R_{\mathrm{LF}}+R_{\mathrm{MF}}\right) / 2} \times 100 \%,
$$

where $R_{\mathrm{LF}}, R_{\mathrm{MF}}$, and $R_{\mathrm{HF}}$ are the ratios between the power at the apex and footpoints in the LF, MF, and HF ranges, respectively. Excess HF power at the loop apex is characterized by positive values of the RD (the HF component power decreases slower than the LF, and MF components when traveling along the loop from the footpoints to the apex), whereas a negative value of the RD would indicate that the HF component decreases faster along the loop than the LF/MF power. The RD value for this particular loop is about $101.56 \%$, indicating a significant excess of HF power at the loop apex.

In addition to the long (green) loop, we have also analyzed the two shorter loops, outlined by the blue and yellow curves in Figure 1(C). We found similar perturbations propagating upward along the loops, with typical herringbone patterns in the corresponding Doppler velocity time-distance diagrams (not shown). However, the behavior of the EHFF in these two loops is of particular interest. Figure 4 shows the corresponding FFT power spectra (averaged and normalized as before) as a function of frequency for these two loops. The FFT power spectrum of the medium loop shows similar behavior as the long loop: the LF FFT power at the apex is lower than the corresponding LF power at the footpoints, but at HFs, the power at the apex is higher than at the footpoints (i.e., in Figure 4(A) the red line (apex) falls below the solid black line (footpoints) at LFs but above the black line at HFs). The effect is not as pronounced though as for the long loop described earlier, with an RD value of about $17.92 \%$. However, the EHFF appears to be absent for the 
(A) Doppler Velocity Original Timeseries

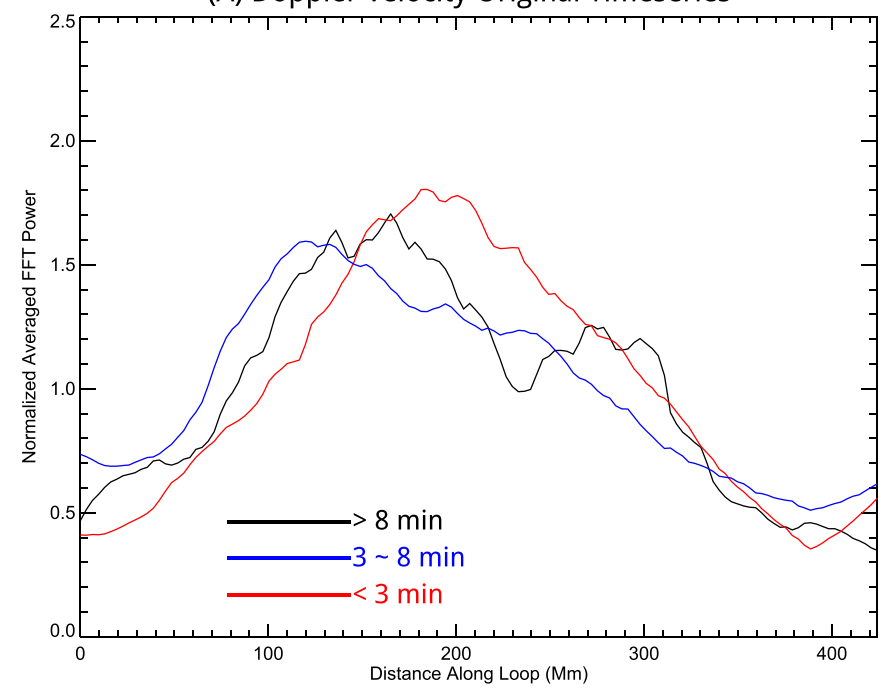

(C) Doppler Velocity Original Timeseries

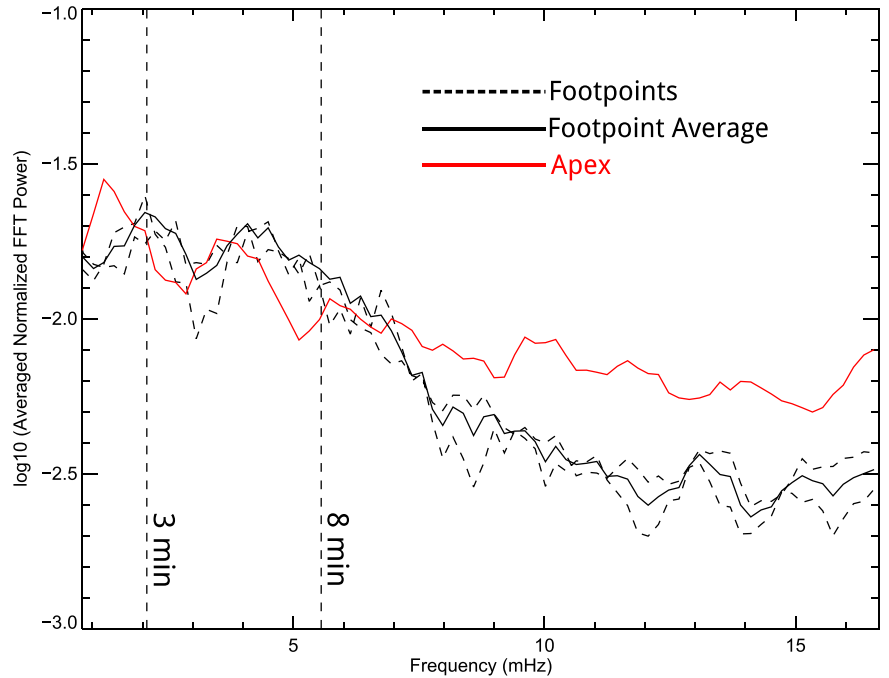

(B) Doppler Velocity Shuffled Timeseries

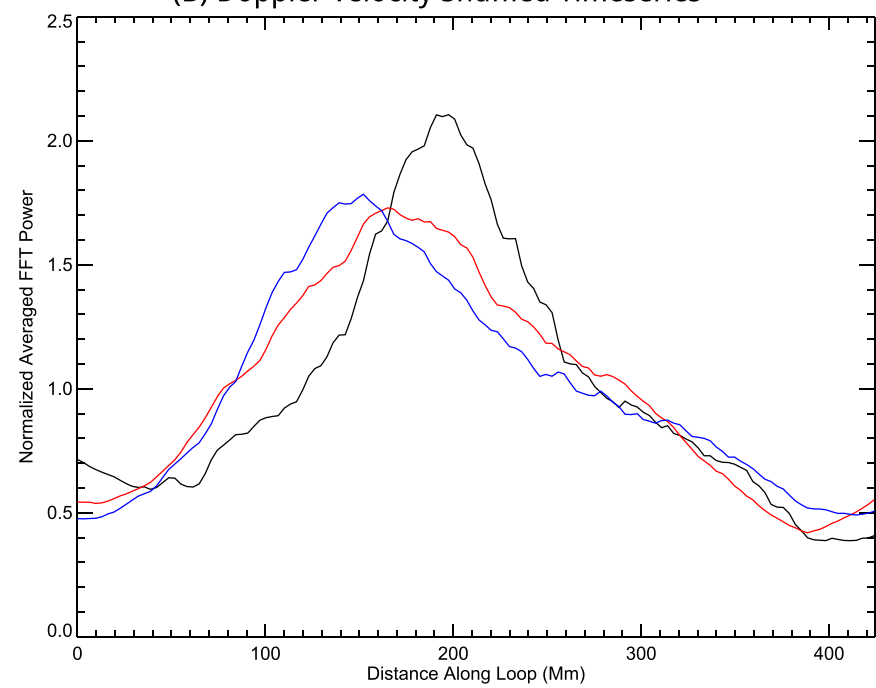

(D) Doppler Velocity Shuffled Timeseries

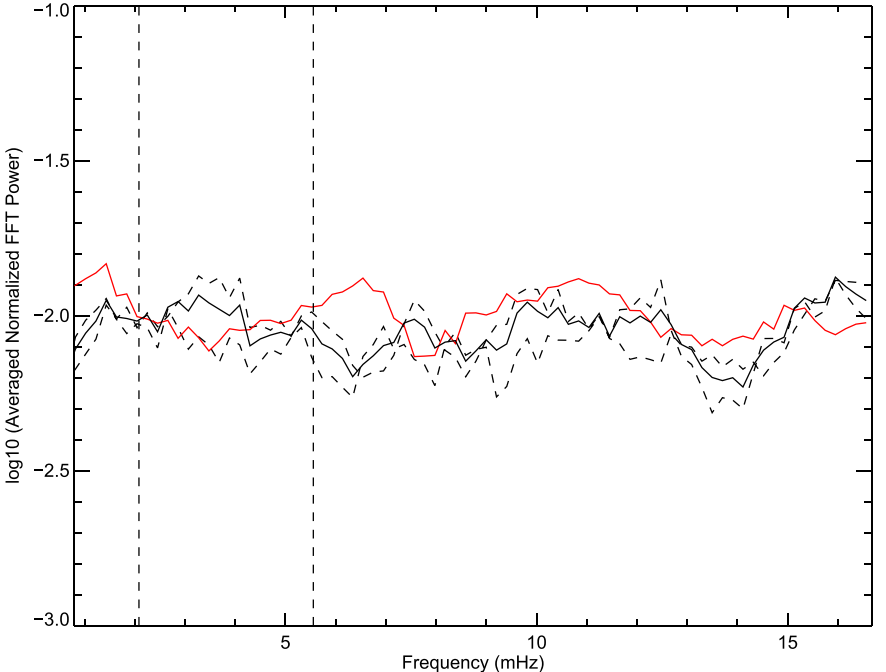

Figure 3. ((A) and (B)) Normalized averaged FFT power at low- $(<3$ minutes, black curve), medium- (between 3 and 8 minutes, blue curve), and high-frequency ( $>8$ minutes, red curve) ranges (referred to as LF, MF, and HF respectively) from the original and reshuffled time series data of the Doppler velocity observation by CoMP on 2013 September 22, respectively. ((C) and (D)) The logarithm of the averaged normalized FFT power at two footpoints (black dashed curves) and the apex (red solid curves) as functions of frequency, based on the original and reshuffled time series data of Doppler velocity, respectively. The black solid curve is the average of the FFT power at two footpoints. The two vertical dashed line represent periods of 3 minutes and 8 minutes, respectively.

(A color version of this figure is available in the online journal.)

shortest loop (Figure 4(C)). The FFT power at the apex is now approximately equal to the FFT power at the footpoints for all frequencies, leading to an RD value of $-16.06 \%$. This intriguing result of different behavior of the HF power in different loops implies that the EHFF phenomenon is not necessarily present in all loops. All three loops studied in this section are located in the same cavity and hence it is likely that they share some properties such as their magnetic field topology (and possibly their magnetic field strength and plasma density). The most clear distinction between them is their lengths, namely $452 \mathrm{Mm}$, $304 \mathrm{Mm}$, and $201 \mathrm{Mm}$, respectively.

\section{STATISTICAL ANALYSIS OF 37 CORONAL LOOPS}

In the previous section we presented the detailed analysis and the comparison of three cavity loops on 2013 September 22. However, since those loops are in the same structure, they may not have much difference in magnetic field topology and strength and plasma density. We have seen that the Alfvénic perturbations propagating along those loops reveal quite different behavior-the EHFF phenomenon can be easily found in the long and medium loops, but not in the short loop. As noted above, we suppose that the value of RD (representing the significance of the EHFF phenomenon in a loop) may be proportional to the loop length. To examine our supposition, we have analyzed 34 more coronal loops using the same methods presented previously. As noted earlier, these loops are chosen if they are clearly (and easily) isolated in the CoMP FOV.

Table 1 provides the measured parameters for these loops: "YMD" is the date of the observation; "P.A." is the position angle of the apex of a loop with respect to due north; " $L$ " is the apparent length of the loop in the plane of the sky; "RD" is the "ratio difference" defined in Section 3; " $V_{\text {phase" }}$ is the Alfvénic perturbation phase speed detected in a loop using the cross-correlation method described in Section 3; and "WD" is the line width difference and represents how much the 
(A) Medium Loop Doppler Velocity Original Timeseries

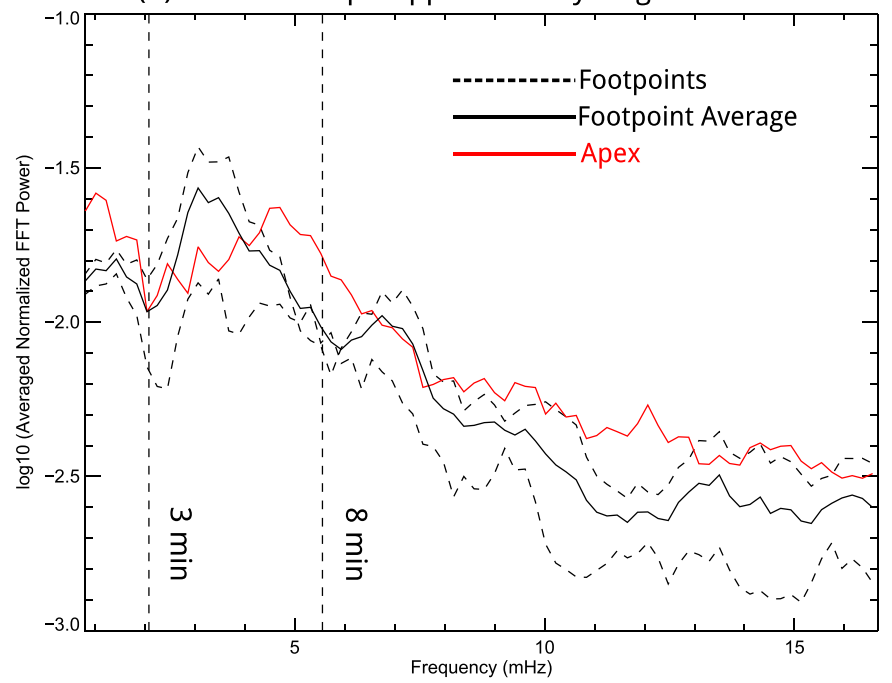

(C) Small Loop Doppler Velocity Original Timeseries

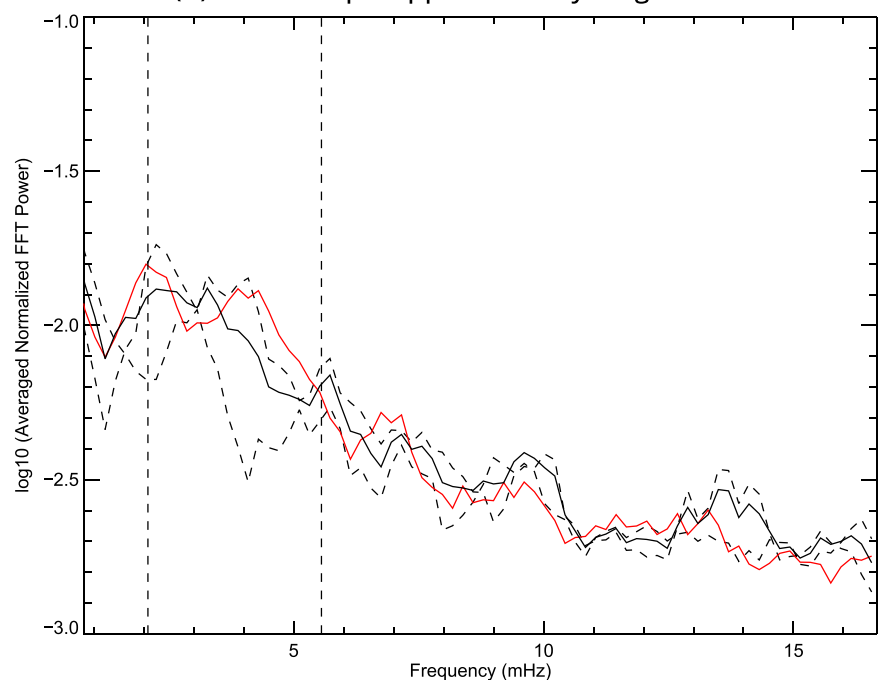

(B) Medium Loop Doppler Velocity Shuffled Timeseries

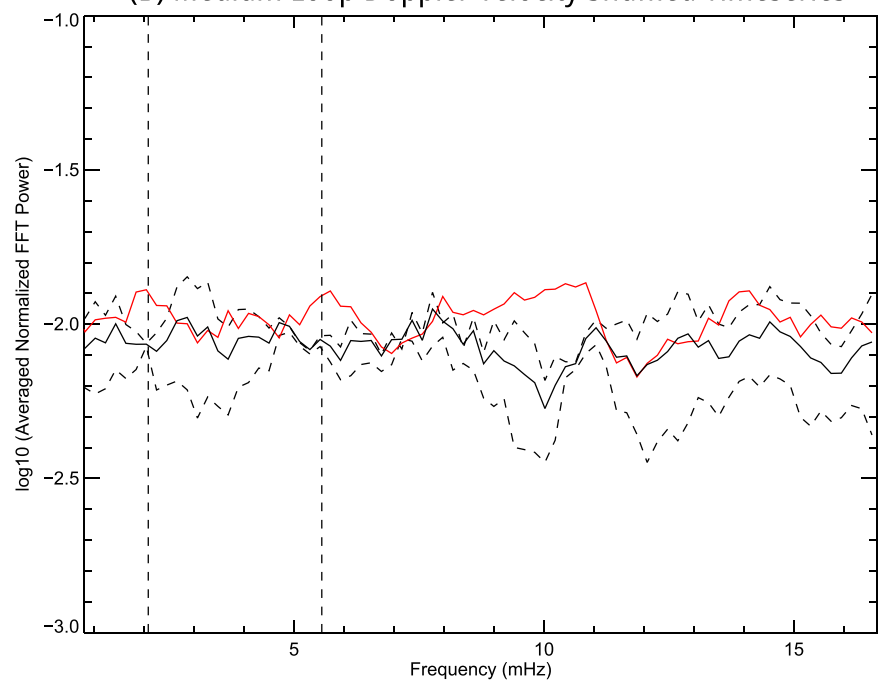

(D) Small Loop Doppler Velocity Shuffled Timeseries

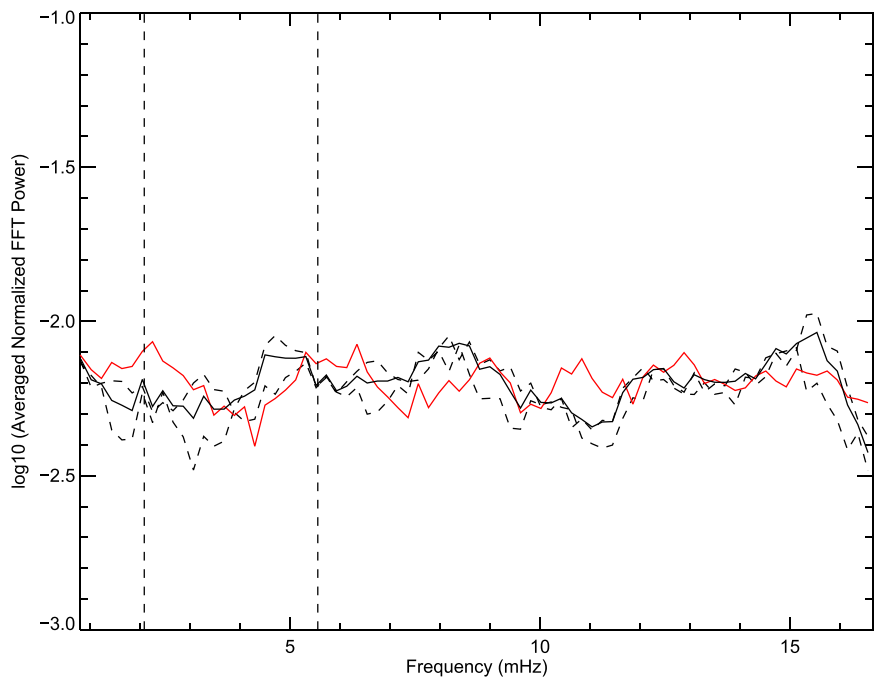

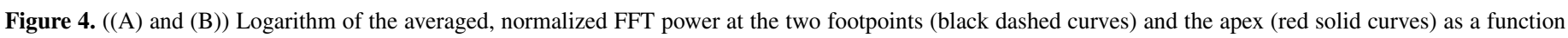

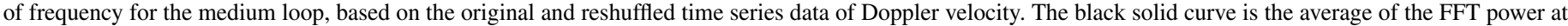

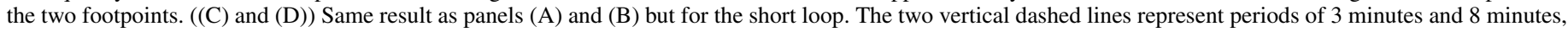
respectively.

(A color version of this figure is available in the online journal.)

average line width increases or decreases at the apex with respect to the footpoints.

The parameter $\lambda$ represents the "characteristic wavelength" of the Alfvénic perturbations propagating in a loop and is defined as follows. We integrate the Doppler velocity FFT power over three narrow wavelength ranges: $1.5 \pm 0.3 \mathrm{mHz}, 3.5 \pm 0.5 \mathrm{mHz}$, and $7.0 \pm 1.0 \mathrm{mHz}$. We then fit the relationship between the three integrated FFT powers and the filter frequencies with a Gaussian-the characteristic frequency is that for which the fitted Gaussian function peaks. The quotient of the wave phase speed and the characteristic frequency we then define as the characteristic wavelength for the loop.

Based on the limited sample in Section 3, we speculated that the value of RD ratio varies with loop length. As the longest loop shows a significant excess of HF FFT power and the shortest loop reveals evidence of roughly equal or even higher damping at the HF part-we believe that the RD metric represents the degree by which the EHFF phenomenon increases with loop length. Plotting the tabulated results in Figure 5(A) would appear to add weight to our early speculation as there appears to be a linear relationship between loop length and the RD.

Figure 5 allows us to analyze different types of coronal loops prevalent in CoMP data: cavity loops, active region loops, and trans-equatorial loops. They are represented by green asterisks, blue diamonds, and red triangles, respectively. It is clear that the RD value grows with the loop length and the relationship between them appears to be linear at least within the loop length range we study of $200-700 \mathrm{Mm}$ and $\mathrm{RD}$ range of $-61 \%$ to $144 \%$. The slopes of the green, blue, and red dashed lines are $0.295,0.287$, and 0.152 , respectively. The solid black line represents the linear fit result of the whole data set with a relatively high cross-correlation factor of about 0.7 , implying a reliable proportional relationship between the loop length and RD. We note that the linear fits can be strongly biased by the two data points enclosed in rectangles-it is not immediately clear what is incorrect or wrong with these measurements. but we exclude them from further analysis. Using a RD of $10 \%$ as a threshold value to indicate excess HF power, the overall linear fit 
Table 1

Statistical Results of 37 Coronal Loops in 2013

\begin{tabular}{|c|c|c|c|c|c|c|c|}
\hline YMD & Type & $\begin{array}{l}\text { P.A. } \\
\left({ }^{\circ}\right)\end{array}$ & $\begin{array}{c}L \\
(\mathrm{Mm})\end{array}$ & $\begin{array}{l}\mathrm{RD} \\
(\%)\end{array}$ & $\begin{array}{c}V_{\text {phase }} \\
\left(\mathrm{km} \mathrm{s}^{-1}\right)\end{array}$ & $\begin{array}{c}\lambda \\
(\mathrm{Mm})\end{array}$ & $\begin{array}{l}\text { WD } \\
(\%)\end{array}$ \\
\hline 20130104 & $\mathrm{CV}$ & 313.47 & 396.56 & -15.01 & $309.45 \pm 19.43$ & 76.73 & 3.67 \\
\hline 20130202 & TE & 90.94 & 299.14 & 21.56 & $332.75 \pm 18.17$ & 79.97 & 5.43 \\
\hline 20130308 & AR & 57.96 & 431.85 & 74.34 & $350.89 \pm 16.62$ & 80.27 & 9.90 \\
\hline 20130308 & AR & 70.02 & 187.41 & -11.13 & $231.86 \pm 34.94$ & 46.73 & 16.61 \\
\hline 20130308 & $\mathrm{TE}$ & 98.88 & 480.31 & 93.37 & $386.82 \pm 31.47$ & 93.82 & 10.33 \\
\hline 20130308 & $\mathrm{CV}$ & 210.45 & 548.42 & 85.46 & $413.91 \pm 23.99$ & 83.78 & 10.64 \\
\hline 20130308 & AR & 260.21 & 544.61 & 90.17 & $386.86 \pm 33.31$ & 82.61 & 26.60 \\
\hline 20130313 & TE & 97.11 & 297.52 & -60.63 & $631.29 \pm 34.23$ & 90.18 & 26.15 \\
\hline 20130313 & AR & 114.03 & 350.61 & -8.78 & $607.34 \pm 33.26$ & 120.50 & 29.63 \\
\hline 20130403 & $\mathrm{TE}$ & 92.13 & 310.59 & 8.13 & $541.18 \pm 41.84$ & 117.74 & 27.28 \\
\hline 20130418 & TE & 267.29 & 664.01 & 13.74 & $571.21 \pm 39.35$ & 124.05 & 8.96 \\
\hline 20130418 & TE & 269.04 & 389.53 & -4.50 & $399.54 \pm 16.20$ & 100.07 & 4.12 \\
\hline 20130502 & TE & 89.29 & 494.53 & 77.24 & $577.09 \pm 23.46$ & 143.72 & 17.10 \\
\hline 20130502 & TE & 91.64 & 263.02 & 4.31 & $608.33 \pm 42.61$ & 154.73 & 2.94 \\
\hline 20130515 & TE & 270.90 & 592.04 & 18.02 & $466.47 \pm 21.55$ & 115.31 & 4.32 \\
\hline 20130623 & AR & 101.67 & 299.20 & -24.93 & $490.29 \pm 29.21$ & 105.90 & 17.22 \\
\hline 20130627 & $\mathrm{CV}$ & 159.39 & 343.49 & 39.79 & $467.83 \pm 27.73$ & 108.10 & 10.88 \\
\hline 20130702 & $\mathrm{CV}$ & 60.32 & 349.22 & 39.52 & $746.17 \pm 39.99$ & 173.94 & 11.48 \\
\hline 20130708 & AR & 235.10 & 573.15 & 74.29 & $309.02 \pm 22.87$ & 56.62 & 20.43 \\
\hline 20130712 & AR & 88.97 & 382.76 & -7.12 & $435.04 \pm 42.98$ & 85.29 & 24.40 \\
\hline 20130715 & $\mathrm{CV}$ & 49.35 & 218.54 & -4.26 & $429.79 \pm 30.48$ & 91.55 & 7.53 \\
\hline 20130914 & $\mathrm{CV}$ & 199.60 & 576.91 & 143.75 & $316.02 \pm 32.02$ & 64.75 & 15.72 \\
\hline 20130914 & TE & 93.67 & 310.76 & 115.36 & $740.61 \pm 29.19$ & 163.35 & 31.48 \\
\hline 20130914 & TE & 94.08 & 217.51 & -34.33 & $619.78 \pm 14.96$ & 129.48 & 8.39 \\
\hline 20130921 & $\mathrm{CV}$ & 59.20 & 468.50 & 65.15 & $416.70 \pm 28.86$ & 94.96 & 8.30 \\
\hline 20130921 & $\mathrm{CV}$ & 60.88 & 323.74 & -7.25 & $537.96 \pm 28.91$ & 129.12 & 2.77 \\
\hline 20130921 & $\mathrm{CV}$ & 60.29 & 204.55 & -12.69 & $334.31 \pm 16.10$ & 79.69 & 7.21 \\
\hline 20130922 & $\mathrm{CV}$ & 57.52 & 452.39 & 101.56 & $460.82 \pm 28.59$ & 108.49 & 6.98 \\
\hline 20130922 & $\mathrm{CV}$ & 57.53 & 304.30 & 17.92 & $621.46 \pm 19.50$ & 151.77 & 3.17 \\
\hline 20130922 & $\mathrm{CV}$ & 57.27 & 201.22 & -16.06 & $506.51 \pm 28.18$ & 122.37 & 8.10 \\
\hline 20130923 & $\mathrm{CV}$ & 53.71 & 373.20 & 24.50 & $588.99 \pm 24.49$ & 134.08 & 7.05 \\
\hline 20130923 & $\mathrm{CV}$ & 54.44 & 186.49 & -11.86 & $304.71 \pm 20.89$ & 70.37 & 2.82 \\
\hline 20131005 & $\mathrm{CV}$ & 202.44 & 426.54 & 73.03 & $250.88 \pm 11.94$ & 58.39 & 8.68 \\
\hline 20131005 & $\mathrm{CV}$ & 296.66 & 445.73 & 4.47 & $452.36 \pm 52.04$ & 102.29 & 4.72 \\
\hline 20131005 & $\mathrm{CV}$ & 323.14 & 265.63 & -11.53 & $366.89 \pm 17.27$ & 94.66 & 7.62 \\
\hline 20131005 & $\mathrm{CV}$ & 324.84 & 446.22 & 79.79 & $361.48 \pm 22.64$ & 86.95 & 3.85 \\
\hline 20131102 & AR & 298.75 & 606.16 & 71.29 & $399.28 \pm 15.97$ & 93.87 & 3.64 \\
\hline
\end{tabular}

Notes. YMD: date. Type: three types of loops, cavity (CV), active region (AR), trans-equatorial (TE). P.A.: position angle of the loop with respect to the arctic pole in units of degree. $L$ : loop length in units of megameter. RD: ratio difference (see the text). $V_{\text {phase}}$ : propagating perturbation phase speed in units of kilometer per second. $\lambda$ : characteristic wavelength, in units of megameter. WD: line width difference (see the text).

(black line) allows us to determine a critical loop length: when a loop is longer than $318 \mathrm{Mm}$, the Alfvénic perturbations appear to damp slower at HFs when propagating from the footpoints to the apex than they do at LFs. The loop studied by De Moortel et al. (2014) has a length of $\sim 480 \mathrm{Mm}$ and do indeed exhibit this behavior.

Alfvénic perturbations propagating along different loops could display different behavior, considering that loops may have different properties (e.g., density, magnetic field strength; Priest 1978). For the propagating waves considered in this study, wavelength is one representation for plasma density, magnetic field strength, and wave frequency. As shown in Figure 5(B), $\mathrm{RD}$ has a proportional relationship with the ratio of the loop length and the characteristic wavelength, a measure we define as the "wave count"- - or the number of waves wholly contained in the loop. Again, we fit the relationship with a linear function and the cross-correlation factor turns out to be larger than 0.5 $(\sim 0.6)$. Following the definition of critical length, we find the value of the critical wave count to be about $3.0 \pm 0.9$ for RD to be large enough (shown as the black dashed lines in Figure 5(B)).

\section{DISCUSSION}

We analyzed 37 clearly isolated CoMP coronal loops. In all cases, Alfvénic perturbations are found propagating along coronal loops. Detailed analysis reveals that these perturbations cause obvious variations in Doppler velocity but not in simultaneous intensity data, implying their incompressible (Alfvénic) nature, as reported in Tomczyk et al. (2007). The phase speed of these perturbations ranges from $250 \mathrm{~km} \mathrm{~s}^{-1}$ to $750 \mathrm{~km} \mathrm{~s}^{-1}$ and their typical period varies from $140 \mathrm{~s}$ to $270 \mathrm{~s}$ (roughly 3-5 minutes) - consistent with those reported in Tomczyk et al. (2007) and Tomczyk \& McIntosh (2009).

Further, time-distance analysis of the Doppler signal in a coronal loop of 2013 September 22 ( $\sim 450 \mathrm{Mm}$ long) revealed a herringbone pattern, indicating perturbations propagating from both footpoints to the apex, similar to Tomczyk \& McIntosh (2009). The bi-directional perturbations have almost the same phase speed and they interact with each other around the apex. No obvious downward propagating perturbations around the footpoints are found, implying that these perturbations 

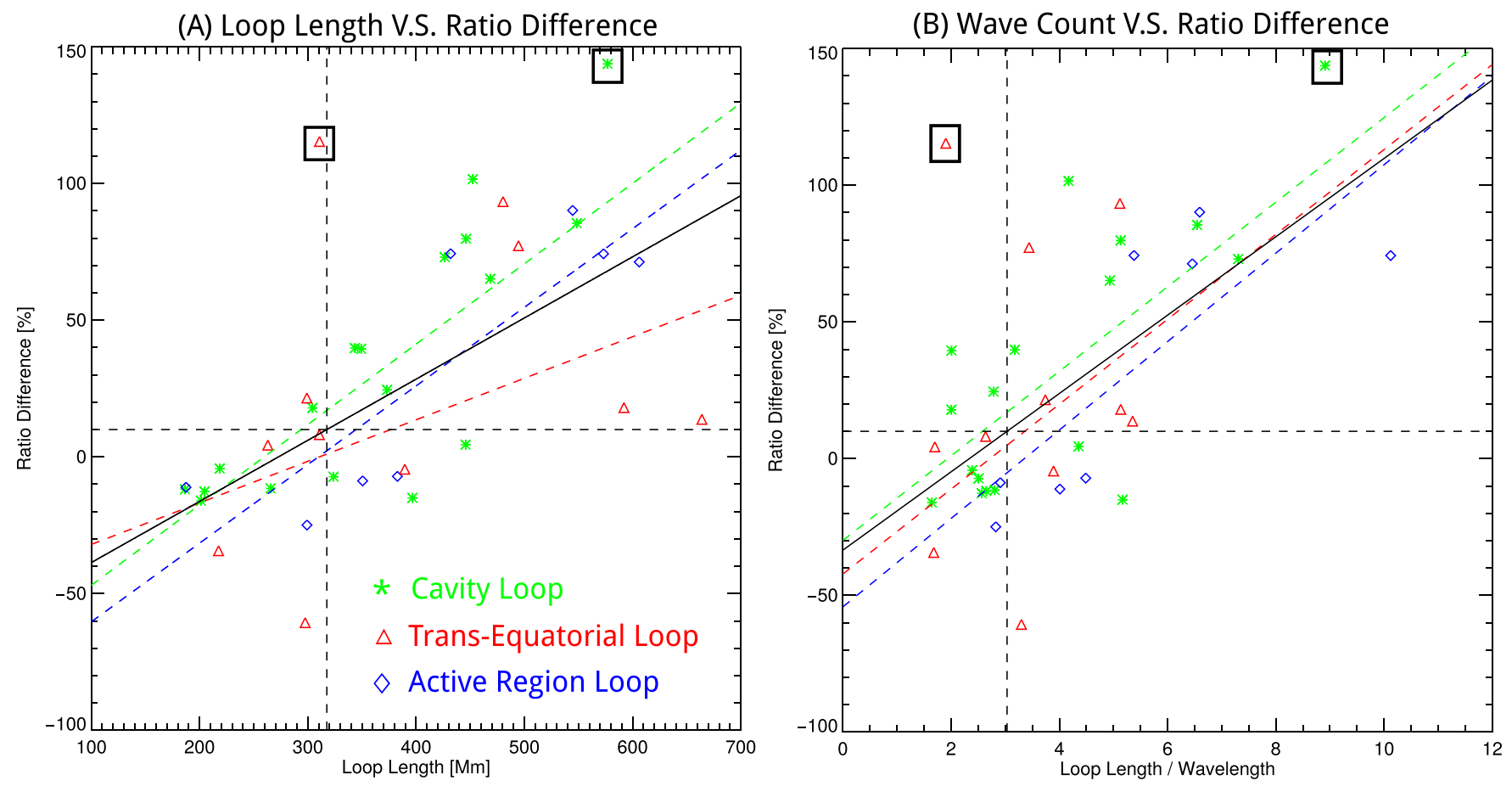

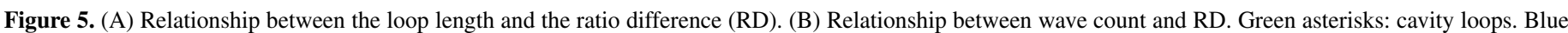

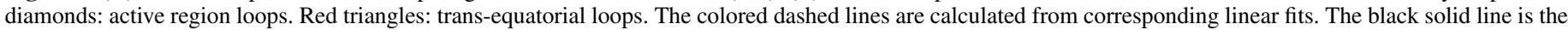
linear fit result for the full data set. The points in black rectangles are found to strongly bias the linear fits and hence they are excluded from further analysis.

(A color version of this figure is available in the online journal.)

damp continuously on approach to, and after passing, the loop apex.

FFT power analysis shows that these perturbations undergo damping when propagating from the footpoints to the apex across the frequency range accessible to CoMP. One possible means of damping these waves is through "mode coupling"-a process inherent to transverse (kink) waves propagating in a loop with an inhomogeneous boundary layer and where the (observed) wave damping occurs due to the transfer of the wave energy from the transverse waves generated at the loop footpoint regions into azimuthal Alfvénic waves as they propagate along the loop (e.g., Melrose 1977; Pascoe et al. 2010, 2013; Hood et al. 2013).

The basic mode coupling process has an inherent frequency dependence: HF waves damp faster than LF waves (e.g., Pascoe et al. 2010; Terradas et al. 2010). This means that we would expect HF FFT power at the loop apex to be lower than observed. Further, in a mode coupling regime, HF perturbations damp faster in higher density loops (which are typically shorter; Terradas et al. 2010). This relationship could explain the different inclinations between the linear fit lines (Figures 5(A) and (B) to cavity loops (green dashed line), active region loops (blue dashed line), and trans-equatorial loops (red dashed line). For example, as the plasma density in active and trans-equatorial regions is likely to be larger than cavities, lower inclinations would be expected. However, further investigation is required to confirm this suggestion.

Given the frequency selectivity in mode coupling, less HF power would be expected at the loop apex compared to lower frequencies. However, this is contrary to what the observations and analysis of the long and medium loops show. As shown in Figure 3, the HF FFT power at the apex of these perturbations is higher than that at the LFs and MFs. Recalling that we defined the RD as a measure of how much the HF power is larger than the
LF/MF power, one would expect the value of RD to be always below 0 if the HF part damps faster (as in the mode coupling model). However, this is not the case and the RD is larger than 0 for the long and medium loops. De Moortel et al. (2014) suggested that the excess of the HF power may be evidence of the onset of turbulence (e.g., van Ballegooijen et al. 2011) caused by the nonlinear interaction of two counter-propagating wave trains. Here, counter-propagating wave trains could be either two wave trains traveling upward from two opposing footpoints, or a wave train interacting with its reflected wave train produced by density variations along the loop.

Our statistical analysis shows that the RD is proportional to the loop length. In other words, the longer the loop is, the more HF power is generated. This seems to intuitively agree with the onset of turbulence due to nonlinear interactions between the oppositely traveling wave trains, if a sufficient number of wavelengths is present along the loop. Figure 5(B) shows the proportional relationship between the wave count (representing the number of wavelengths along the loop) and the RD. This plot reveals that the more wave lengths there are along the loop, the more HF power is generated-this again would tend to support the premise that turbulence is present.

If turbulence is present, the line width of optically thin lines like those observed by CoMP would be expected to increase at the apex. However, many other effects influence line widths. For example, gravitational stratification and lineof-sight superposition can increase the observed line widths (McIntosh \& De Pontieu 2012), whereas damping of waves has the opposite effect. This complexity makes studying the direct relationship between the wave count and the WD (defined in Section 4 and shown in Table 1) meaningless. To subtract the influence of stratification, damping and some other effects that may change the line width with height, we calculate the difference between the average line width at the height of the 


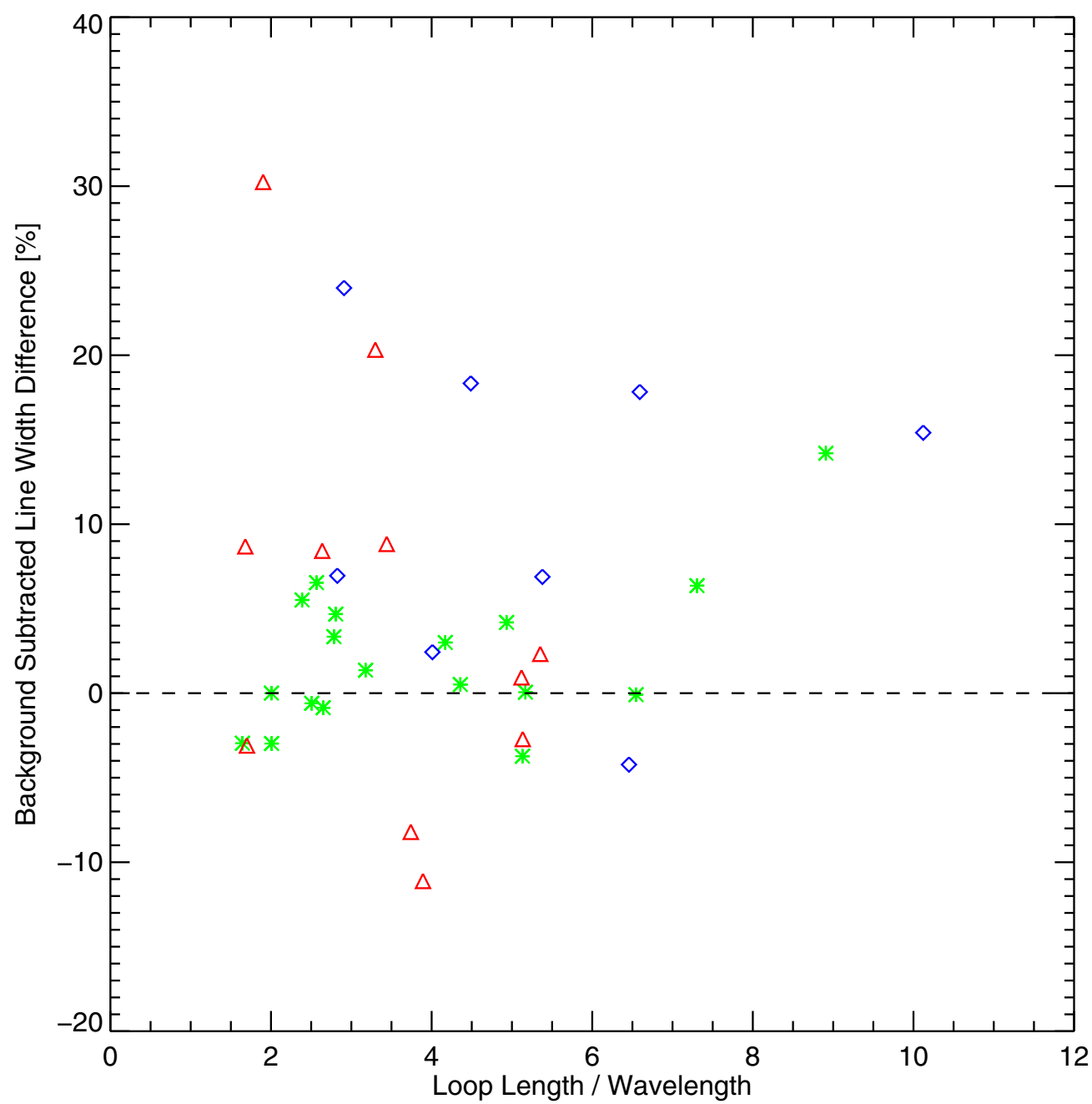

Figure 6. Relationship between the wave count and the background-subtracted line width difference. Green asterisks: cavity loops. Blue diamonds: active region loops. Red triangles: trans-equatorial loops.

(A color version of this figure is available in the online journal.)

apex and the footpoints over the whole time sequence with a $1^{\circ}$ angular spacing around the Sun as the line width difference of the background ("WDB"). Figure 6 shows the relationship between the wave count and the difference between WD and WDB. We see that $\sim 70 \%$ of the points lie above 0 (the horizontal dashed line) possibly indicating that the line broadening at the majority of the loop apexes sampled is larger than may be expected via waves propagating through a complex structural superposition. However, such superposition likely will not change the loop lengths or wave counts, possibly indicating that the distribution of the points in Figure 6 could be a signature of turbulence in coronal loops.

\section{CONCLUSION}

In this paper, following the work by De Moortel et al. (2014), we performed a detailed analysis of three cavity loops observed on 2013 September 22. Doppler velocity perturbations with phase speeds around $640 \mathrm{~km} \mathrm{~s}^{-1}$ propagating from the loop footpoints are found in the corresponding time-distance diagrams without any simultaneous density variations. An excess of HF power (EHFF) is found in the FFT power spectrum of the two long loops and not in that of the shortest loop. This EHFF phenomenon might be tentative evidence for the onset of Alfvénic turbulence. Further statistical analysis on 37 clearly isolated loops shows a relationship between the loop length and the RD (a measure of the excess HF at the apex), in agreement with the assumption of turbulence, as a longer traveling distance could lead to more (nonlinear) interactions between oppositepropagating wave trains. Linear fits reveal a loop length of at least about $318 \mathrm{Mm}$ for the EHFF phenomenon to be present.

The proportional relationship between the wave count (how many wavelengths there are along a loop) and the RD tends to support the presence of Alfvénic turbulence. The critical wave count of 3.0 for the excess HF (EHFF) to be present is consistent with (the onset of) turbulence. Finally, we have explored the relationship between the wave count and the background subtracted line width. The weakly proportional relationship between them again supports the onset of Alfvénic turbulence in coronal loops.

In this study, we have presented statistical evidence for the onset of Alfvénic turbulence in coronal loops. However, the relatively low spatial resolution and signal-to-noise ratio of CoMP data prevent us from performing a more detailed analysis and finding more direct evidence of turbulence in those loops. We hope that future, more detailed observations using instruments with higher spatial resolution and signal-to-noise, combined with numerical simulations will help improve our 
understanding of coronal turbulence and any potential impact on coronal heating.

J.L. is a student visitor at HAO. J.L. is supported by the Chinese Scholarship Council (CSC 201306340034) and also supported by grants from NSFC 41131065, 41121003, 973 Key Project 2011CB811403 and CAS Key Research Program KZZD-EW-01-4. J.L. also thanks his advisor in China, Dr. Yuming Wang. NCAR is sponsored by the National Science Foundation. CoMP data can be found at http:// mlso.hao.ucar.edu/. We acknowledge support from NASA contracts NNX08BA99G, NNX11AN98G, NNM12AB40P, NNG09FA40C (IRIS), and NNM07AA01C (Hinode). The research leading to these results has also received funding from the European Commission Seventh Framework Programme (FP7/ 2007-2013) under the grant agreement SOLSPANET (project No. 269299, www.solspanet.eu/solspanet).

\section{REFERENCES}

Antolin, P., \& Verwichte, E. 2011, ApJ, 736, 121

Aschwanden, M. J., Fletcher, L., Schrijver, C. J., \& Alexander, D. 1999, ApJ, 520,880

Asgari-Targhi, M., \& van Ballegooijen, A. A. 2012, ApJ, 746, 81

Cirtain, J. W., Golub, L., Lundquist, L., et al. 2007, Sci, 318, 1580

Cranmer, S. R., \& van Ballegooijen, A. A. 2005, ApJS, 156, 265

De Moortel, I., McIntosh, S. W., Threlfall, J., Bethge, C., \& Liu, J. 2014, ApJL, 782, L34

De Moortel, I., \& Nakariakov, V. M. 2012, RSPTSA, 370, 3193

De Moortel, I., \& Pascoe, D. J. 2012, ApJ, 746, 31

De Pontieu, B., McIntosh, S. W., Carlsson, M., et al. 2007, Sci, 318, 1574

Erdélyi, R., \& Fedun, V. 2007, Sci, 318, 1572
Goossens, M., Van Doorsselaere, T., Soler, R., \& Verth, G. 2013, ApJ, 768, 191 He, J., Marsch, E., Tu, C., \& Tian, H. 2009, ApJL, 705, L217

Hood, A. W., Ruderman, M., Pascoe, D. J., et al. 2013, A\&A, 551, A39

Liu, J., Zhou, Z., Wang, Y., et al. 2012, ApJL, 758, L26

McIntosh, S., Pontieu, B., \& Tomczyk, S. 2008, SoPh, 252, 321

McIntosh, S. W. 2012, SSRv, 172, 69

McIntosh, S. W., \& De Pontieu, B. 2012, ApJ, 761, 138

McIntosh, S. W., de Pontieu, B., Carlsson, M., et al. 2011, Natur, 475, 477

Melrose, D. B. 1977, AuJPh, 30, 495

Morton, R. J., \& McLaughlin, J. A. 2013, A\&A, 553, L10

Nakariakov, V. M., \& Verwichte, E. 2005, LRSP, 2, 3

Okamoto, T., Tsuneta, S., Berger, T., et al. 2007, Sci, 318, 1577

Osterbrock, D. E. 1961, ApJ, 134, 347

Oughton, S., Matthaeus, W. H., Dmitruk, P., et al. 2001, ApJ, 551, 565

Parker, E. N. 1991, ApJ, 372, 719

Parnell, C. E., \& De Moortel, I. 2012, RSPTA, 370, 3217

Pascoe, D. J., Hood, A. W., De Moortel, I., \& Wright, A. N. 2013, A\&A, 551, A40

Pascoe, D. J., Wright, A. N., \& De Moortel, I. 2010, ApJ, 711, 990

Priest, E. R. 1978, SoPh, 58, 57

Smartt, R. N., Dunn, R. B., \& Fisher, R. R. 1981, Proc. SPIE, 288, 395

Suzuki, T. K., \& Inutsuka, S.-i. 2005, ApJL, 632, L49

Terradas, J., Goossens, M., \& Verth, G. 2010, A\&A, 524, A23

Threlfall, J., De Moortel, I., McIntosh, S. W., \& Bethge, C. 2013, A\&A, 556, A 124

Tomczyk, S., Card, G. L., Darnell, T., et al. 2008, SoPh, 247, 411

Tomczyk, S., \& McIntosh, S. W. 2009, ApJ, 697, 1384

Tomczyk, S., McIntosh, S. W., Keil, S. L., et al. 2007, Sci, 317, 1192

Ulmschneider, P., \& Musielak, Z. 1990, in ASP Conf. Ser. 9, Cool Stars, Stellar Systems, and the Sun, ed. G. Wallerstein (San Francisco, CA: ASP), 116

van Ballegooijen, A. A., Asgari-Targhi, M., Cranmer, S. R., \& DeLuca, E. E. 2011, ApJ, 736, 3

Van Doorsselaere, T., Nakariakov, V. M., \& Verwichte, E. 2008, ApJL, 676, L73

Verdini, A., Velli, M., Matthaeus, W. H., Oughton, S., \& Dmitruk, P. 2010, ApJL, 708, L116

Wright, A. N., \& Garman, A. R. 1998, JGR, 103, 2377 